panel included: p16, p53, MLH1, MSH2, MSH6, PMS2, PD-L1, CD3, HER2/neu, ER, PR, EGFR, VEGF and CD31. The reactions were evaluated on qualitative and semi-quantitative scale. Generalized Linear Model (GLM) and Cluster analysis were performed in $\mathrm{R}$ statistical environment. A distance plot compared the IHC panel of $\mathrm{T}$ with the correspondent $\mathrm{N}$.

Result(s)* Mismatch repair proteins (MMR), ER, PR and HER2/neu were excluded from data analysis because of homogeneous expression in all samples. Group A: the p16positive expression (surrogate of HPV-dependent pathway) was significantly higher $(20.8 \%$ vs $6.2 \%, p=0.04)$. Group B: PD-L1-positive and high EGFR expression were found respectively in $77.1 \%$ and $97.9 \%$ patients, ( $T$ and/or $\mathrm{N}$ ). Overall, p16-negative tumors showed a higher PD-L1 expression $(60.9 \%$ vs. $50.0 \%)$. In both groups tumoral immune infiltration (CD3 expression), was mainly moderate/intense $(80 \%$ vs. 95\%). VEGF showed strong/moderate-diffuse expression in $13.9 \%$ of $\mathrm{T}$ samples. CD31 was used to study tumoral micro vessel density (MVD) with no difference between Group A and Group B. p53 and PD-L1 showed a significant association with nodal metastasis. Odds ratio (OR) for p53 mutation was 4.26 (CI 95\% $=1.14-15.87, \mathrm{p}=$ 0.03 ); OR for PD-L1 positivity was 2.68 (CI 95\% $=1.0-$ 7.19, $\mathrm{p}<0.05)$.

The cluster analysis identified 3 and 4 sub-groups of molecular profiles respectively in Group A and B, with no different prognosis. Moreover, the molecular profile of each $\mathrm{N}$ and corresponding $\mathrm{T}$ diverged significantly in $18 / 41$ (43.9\%) cases.

Conclusion* These results support a potential role of immune checkpoint inhibitors and anti-EGFR drugs in a subset of patients with VSCC, especially with worse prognosis (metastatic, HPV-independent). It is mandatory to repeat the panel in the metastatic site to identify changes of marker expression.

\section{UPDATE OF THE GROSNAPET STUDY: ON THE WAY TO OVERCOME SENTINEL NODE LIMITS IN VULVAR CANCER}

\begin{abstract}
${ }^{1} \mathrm{SM}$ Fragomeni* ${ }^{*}{ }^{2} \mathrm{~A}$ Collarino, $2,3 \mathrm{~V}$ Rufini, ${ }^{1} \mathrm{~A}$ Federico, ${ }^{4} \mathrm{~F}$ Inzani, ${ }^{1} \mathrm{G}$ Corrado, ${ }^{5,6} \mathrm{~S}$ Gentileschi, ${ }^{7} \mathrm{~L}$ Tagliaferri, ${ }^{8} \mathrm{D}$ Verri, ${ }^{9} \mathrm{~A}$ Zampolini Faustini, ${ }^{2} \mathrm{~A}$ Florit, ${ }^{1,9} \mathrm{~A}$ Fagotti, ${ }^{1,9} \mathrm{G} \mathrm{Scambia},{ }^{8,9} \mathrm{G}$ Garganese. ${ }^{1}$ Fondazione Policlinico Universitario A. Gemelli IRCCS, Unità di Ginecologia Oncologica, Dipartimento Scienze della Salute della Donna, del Bambino e di Sanità Pubblica, Rome, Italy; ${ }^{2}$ Fondazione Policlinico Universitario A. Gemelli IRCCS, Unità di Medicina Nucleare, Dipartimento di diagnostica per Immagini, Radioterapia Oncologica ed Ematologia, Rome, Italy; ${ }^{3}$ Università Cattolica del Sacro Cuore, Unità di Medicina Nucleare, Dipartimento di diagnostica per Immagini, Radioterapia Oncologica ed Ematologia, Rome, Italy; ${ }^{4}$ Fondazione Policlinico universitario Agostino Gemelli IRCCS, Unità di GinecoPatologia e Patologia Mammaria, Dipartimento Scienze della Salute della Donna, del Bambino e di Sanità Pubblica, Rome, Italy; ${ }^{5}$ Università Cattolica del Sacro Cuore, Istituto di Clinica Chirurgica, Rome, Italy; ${ }^{6}$ Fondazione Policlinico Universitario A. Gemelli IRCCS, Unità di Chirurgia Plastica, Dipartimento Scienze della Salute della Donna, del Bambino e di Sanità Pubblica, Rome, Italy; ${ }^{7}$ Fondazione Policlinico Universitario A. Gemelli, IRCCS, Unità Operativa Complessa di Radioterapia, Dipartimento di Scienze Radiologiche, Radioterapiche ed Ematologiche, Rome, Italy; ${ }^{8}$ Mater Olbia Hospital, Gynecology and Breast Care Center, Olbia, Italy: ${ }^{9}$ Università Cattolica del Sacro Cuore, Istituto di Clinica Ostetrica e Ginecologica, Rome, Italy
\end{abstract}

\subsection{6/ijgc-2021-ESG0.633}

Introduction/Background* Sentinel node biopsy (SNB) is the standard of care for vulvar cancer patients clinically N0 (cN0) at preoperative assessment, with unifocal primary tumor, $<4$ $\mathrm{cm}$, not previously excised nor subjected to neoadjuvant treatment. The cN0 patients unsuitable for these strict criteria currently undergo to radical lymphadenectomy, resulting unnecessary in the $70 \%$ of cases, due to a negative final histology. In our previous prospective trial (GroSNaPET study) we performed in this subgroup of patients the SNB followed by standard lymphadenectomy, demonstrating safety and accuracy of SLN (73 groins enrolled). In this report on the extended series including a total of 111 groins, we update the follow up data.

Methodology According to the Grosnapet study design, lymph node status was assessed by pre-operative PET/CT scan and cN0 patients were enrolled on the base of the following criteria: a) Tumor $>4 \mathrm{~cm}$, b) Multifocal tumors, c) previous complete excision; d) contralateral nodal involvement, e) previous RTCT treatment or $\mathrm{f}$ ) vulvar recurrence. Vulvar surgery was performed according to current recommendations, as appropriate. Sentinel lymph node was detected by radiotracer and blue dye to reach the maximum detection rate. Radical lymphadenectomy was always provided after SNB, according to the standard of care. Both PET/CT scan and SNB were compared to final pathology report. Patients were followed up quarterly, undergoing clinical visit, groin ultrasound and PET/CT scan.

Result(s)* During the study period, 72 patients were considered eligible for a total of 111 groins included. Median patient's age was 73yrs. Histopathology revealed 16 (14.4\%) groins with metastatic sentinel nodes (SLNs). Only one case had further involved non SLNs. Overall, 18/274 (6.5\%) SLN excised were positive at histology. Median metastasis diameter was 4.8 (1.5-12). One false negative SN was identified (NPV 99\%). PET/CT showed an NPV of 92\%. After a median follow-up of 38 months (range 1-97 months), 19 recurrences and 6 deaths were registered. The 3-year disease-free survival (DFS) was $72.6 \%$.

Conclusion* This is the largest series that strongly support the use of SNB in cN0 patients currently excluded. A careful preoperative study could safely select eligible patients. Further validation is advisable.

\section{VULVAR CANCER TREATMENT BETWEEN 2010 AND 2019: THE EXPERIENCE OF A SINGLE ROMANIAN CANCER CENTER}

${ }^{1} \mathrm{~V}$ Galatan*, ${ }^{1} \mathrm{~N}$ Todor, ${ }^{1,2} \mathrm{AT}$ Kirsch-Mangu, ${ }^{1,2} \mathrm{D}$ Pop, ${ }^{1} \mathrm{Cl}$ Trifan, ${ }^{1} \mathrm{C}$ Ordeanu, ${ }^{1} \mathrm{R}$ Bodea, ${ }^{1} \mathrm{C}$ Pop, ${ }^{1,2} \mathrm{O}$ Coza, ${ }^{1,2}$ VM Nagy, ${ }^{1,2}$ P Achimaş Cadariu, ${ }^{1,2} \mathrm{FL}$ Ignat, ${ }^{1,2} \mathrm{DT}$ Eniu, ${ }^{1,3} \mathrm{AP}$ Trăilă, ${ }^{1,2} \mathrm{G}$ Lazar, '\$ Hica, ${ }^{1,2} \mathrm{AC}$ Rancea, ${ }^{1,2} \mathrm{IC}$ Lisencu, ${ }^{1,2} \mathrm{IC} \mathrm{Vlad},{ }^{3} \mathrm{~A}$ Trăilă. ${ }^{1} T$ The Oncology Institute "Prof. Dr. Ion Chiricuță" Cluj-Napoca, Cluj-Napoca, Romania; ${ }^{2}$ University of Medicine and Farmacy "Iuliu Hatieganu" Cluj-Napoca, Romania, Cluj Napoca, Romania; ${ }^{3}$ General Hospital "Humanitas" Cluj-Napoca, Romania, Surgery, Cluj Napoca, Romania

\subsection{6/ijgc-2021-ESGO.634}

Introduction/Background* The purpose of this study was to evaluate 5-year overall survival (OS), disease free survival (DFS) and local control (LC) for patients diagnosed with primary vulvar cancer and treated at Institute of Oncology "Prof. Dr.Ion Chiricuta" Cluj-Napoca, Romania.

Methodology Between 2010 and 2019 a number of 306 patients with vulvar cancer were treated in our institution, from which we included in this retrospective study 233 patients with squamous cell vulvar cancer; based on FIGO staging: $19(8.2 \%)$ patients were stage 0,115 (49.4\%) stage I, $17(7.3 \%)$ stage II, $66(28.3 \%)$ stage III and $16(6.8 \%)$ 


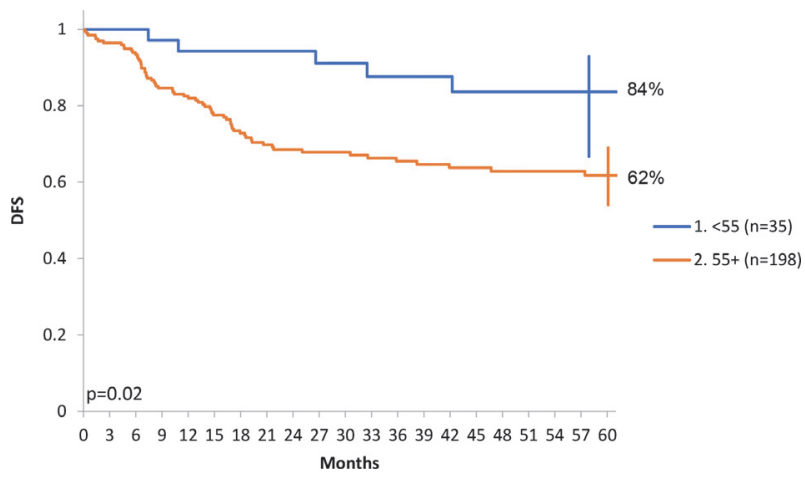

Abstract 1081 Figure 1 Disease free survival based on age groups

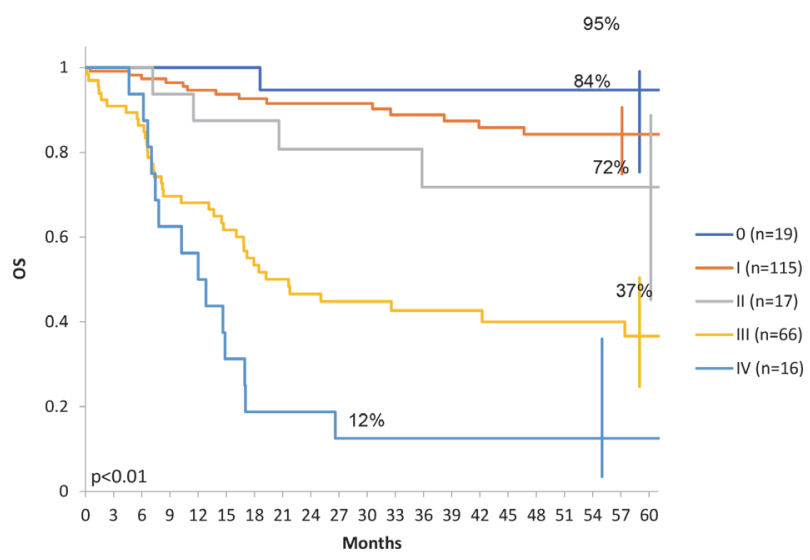

Abstract 1081 Figure 2 Overall survival FIGO stages

stage IV. 146 patients (62.7\%) underwent exclusive surgery, $70(30 \%)$ had surgery combined with adjuvant treatment and $17(7.3 \%)$ with palliative treatment. After these therapeutic modalities patients were followed for a median of 47.1 months (minimum 4.5 months and maximum 122.4 months). Result(s)* The median age was 68 years (minimum of 28 years and maximum of 89 years) and median number of cases per year was 23.3. The OS by stage of disease was $95 \%$, $84 \%, 72 \%, 37 \%$ respectively $12 \%$ for stage 0 , I, II, III respectively IV $(\mathrm{p}<0.01)$. The OS for patients treated with exclusive surgery, surgery combined with adjuvant treatment respectively palliative treatment was 74\%, 61\% respectively $12 \% \quad(\mathrm{p}<0.01) .150 \quad(64.4 \%)$ patients had lymphadenectomy with a median number of 8 lympnodes (minimum 0 , maximum 47); patient with negative lymph nodes and without extracapsular effraction (EC) had OS, DFS and LC significantly improved $(\mathrm{p}<0.01)$. DSF for stage I respectively II was $67 \%$ respectively $62 \%$; LC for stage I respectively stage II was $68 \%$ and $62 \%(\mathrm{p}<0.01)$. Patients with exclusive surgery and surgery combined with adjuvant treatment, had a DSF and LC significantly increased $(\mathrm{p}<0.01)$ in comparison with palliative treatment.

Conclusion* Squamous cell vulvar cancer is a rare cancer in our institution. Surgery remains the best treatment modality for early stages of the disease (stage I and II) and gives high survival rate, DFS and LC, among negative lymph nodes involvement and without extracapsular effraction. For better outcomes there is need for treatment personalization according to new international protocols.

\section{A NATIONAL RETROSPECTIVE STUDY ON EFFICACY OF ADJUVANT (CHEMO)RADIOTHERAPY FOR VULVAR CANCER}

${ }^{1} \mathrm{C}$ Casà ${ }^{*},{ }^{2} \mathrm{G}$ Macchia, ${ }^{3} \mathrm{M}$ Ferioli, ${ }^{1} \mathrm{~V}$ Lancellotta, ${ }^{2} \mathrm{M}$ Boccardi, ${ }^{4} \mathrm{~A}$ Cerrotta, ${ }^{5} \mathrm{~F}$ Tortoreto, ${ }^{6}$ E Ippolito, ${ }^{7} \mathrm{C}$ Laliscia, ${ }^{8} \mathrm{~A}$ Huscher, ${ }^{9} \mathrm{JDI}$ Muzio, ${ }^{3} \mathrm{AG}$ Morganti, ${ }^{10} \mathrm{R}$ Lazzari, ${ }^{11} \mathrm{G}$ Ferrandina, ${ }^{1} \mathrm{~L}$ Tagliaferri. ${ }^{1}$ Fondazione Policlinico Universitario A. Gemelli IRCCS, Dipartimento di Diagnostica per Immagini, Radioterapia Oncologica ed Ematologia, Rome, Italy; ${ }^{2}$ Gemelli Molise Hospital - Università Cattolica del Sacro Cuore, Radiation Oncology Unit, Campobasso, Italy; ${ }^{3}$ RRCCS Azienda Ospedaliero-Universitaria di Bologna - Alma Mater Studiorum University of Bologna, Radiation Oncology, Department of Experimental, Diagnostic and Specialty Medicine - DIMES, Bologna, Italy; ${ }^{4}$ Fondazione IRCCS Istituto Nazionale dei Tumori, Radiotherapy Unit, Milan, Italy; ${ }^{5}$ Ospedale San Pietro Fatebenefratelli, Radiotherapy Unit, Rome, Italy; ${ }^{6}$ Università Campus Bio-Medico, Radiation Oncology, Rome, Italy; 'University of Pisa, Department of New Technologies and Translational Research, Division of Radiation Oncology, Pisa, Italy; ${ }^{8}$ Fondazione Poliambulanza, U. O. di Radioterapia Oncologica "Guido Berlucchi", Brescia, Italy; ${ }^{9}$ A.O.U "Città della Salute e della Scienza", Dipartimento di Oncologia P.O. S. Anna - SS Radioterapia, Turin, Italy; ${ }^{10}$ IEO European Institute of Oncology IRCCS, Division of Radiotherapy, Milan, Italy; ${ }^{11}$ Fondazione Policlinico Universitario A. Gemelli, IRCCS - Università Cattolica del Sacro Cuore, Unità Operativa Complessa Ginecologia Oncologica, Dipartimento per la Salute della Donna e del Bambino e della Salute Pubblica, Rome, Italy

\subsection{6/ijgc-2021-ESG0.635}

Introduction/Background* Vulvar cancer (VC) is a rare gynaecologic malignancy. The study aims to evaluate in a retrospective, multicentred, real-world dataset of patients affected by VC efficacy and safety of adjuvant (chemo)radiotherapy (aRT), focusing especially to the treatment prescription among participant centers joining the Observational itaLian stuDy on vuLvar cAncer adjuvant raDiotherapY (OLDLADY) project endorsed by the GYN study group of the AIRO (Italian Association of Radiotherapy and Clinical Oncology).

Methodology Patients affected by VC and treated by eRT from January 2010 to December 2018 in Nine Italian radiotherapy centers were retrospectively included and clinical data were collected, analysing tumour staging, treatment prescription, clinical and toxicity outcomes.

Result(s)* The multicentred retrospective cohort was composed by 181 patients (patient populations shown in table 1), with a median age of 71.5 years (range 17-90). A considerable heterogeneity was found in the treatment prescription, radiotherapy doses and concomitant chemotherapy schedules. The most relevant acute toxicity revealed for the patient cohort was skin toxicity $(22.1 \%)$; instead, the rate of severe chronic lymphoedema was $1.1 \%$. After a median follow-up of 30 months (range 1-185), the 2-year actuarial local control rate (LC), the 2-year disease free survival rate (DFS) and the overall survival rate (OS) were 57.2\%, 49.4\%, and 55.1\%, respectively. Kaplan-Meier curves were computed and reported in figure 1. Conclusion* A diverse spectrum of radiotherapy options to exclusively treat patients affected by VC was reported. To achieve higher treatment standardisation, in terms of prescribed doses and volumes, this study suggest further cooperative prospective studies.
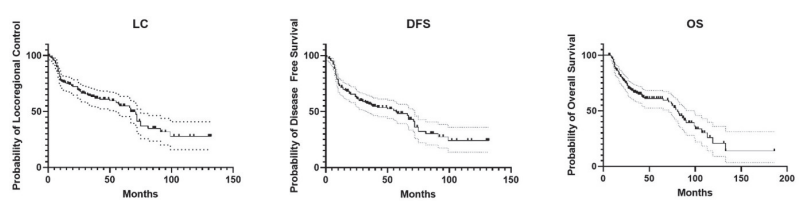

Abstract 1103 Figure 1 Kaplan meier curves for LC, DFS and OS 